\title{
Prevalência de aderências intra-uterinas após aspiração manual a vácuo para tratamento de abortamento
}

\author{
Prevalence of intrauterine adhesions after manual vacuum aspiration for the tratment of abortion \\ Carlos André de Carvalho Godoy ${ }^{1}$, Maria Rachel Aguiar Cordeiro ${ }^{2}$, Rodrigo Serrano ${ }^{3}$, \\ Hélio de Lima Ferrreira Fernandes Costa ${ }^{4}$
}

\section{RESUMO}

Objetivo: avaliar a prevalência de aderências intra-uterinas após aspiração manual a vácuo para tratamento de abortamento. Métodos: estudo prospectivo, descritivo, com comparação de grupos, realizado no período de novembro de 2000 a agosto de 2001, em 80 mulheres de 15 a 48 anos internadas em hospital público para tratamento de qualquer tipo de abortamento. Aaspiração manual a vácuo consistiu na retirada do conteúdo uterino por sucção com seringa de Karman conectada a cânulas plásticas. As aderências intrauterinas foram rastreadas em todas as pacientes, por meio de histeroscopia, realizada 40 a 60 dias após a aspiração. Para análise dos resultados foram utilizados o teste exato de Fisher, ANOVA e estimativa do risco de prevalência, quando indicados. Resultados: a histeroscopia identificou 13 pacientes com aderências intra-uterinas (prevalência de 16,3\%), sendo a maioria delas de constituição mucosa ( 9 de 13), gravidade mínima (11 de 13)e localização lateral no útero ( 5 de 13). Foram mais freqüentes nas mulheres com mais de 8 semanas de gravidez $(24,4 v s 3,7 \%$, risco de prevalência de 6,6) e naquelas com abortamento retido $(41,7 v s 12,1 \%$, risco de prevalência de 3,54). Conclusão: a prevalência de aderências intra-uterinas após aspiração manual a vácuo foi $16,3 \%$. Aborto retido e idade gestacional avançada parecem estar associados à ocorrência das aderências.

PALAVRAS-CHAVE: Aderências; Doenças uterinas; Aborto; Curetagem a vácuo/métodos

\section{ABSTRACT}

Purpose: to evaluate the prevalence of intrauterine adhesions after manual vacuum aspiration for the treatment of abortion. Methods: a prospective, descriptive study, with group comparison was carried out between November 2000 and August 2001 on 80 women between 15 and 48 years old, admitted to a public hospital for abortion care. Manual vacuum aspiration consisted of extracting the uterine content by suction using a Karman syringe connected to plastic cannulas. Intrauterine adhesions were screened by hysteroscopy, performed 40-60 days after the aspiration. Statistical analysis was performed using Fisher exact test, ANOVA and estimation of prevalence risk, when indicated. Results: hysteroscopy identified intrauterine adhesions in 13 patients (prevalence $=16.3 \%$ ), most of them of the mucosal type ( 9 of 13$)$, minimal severity (11 of 13) and lateral location in the uterus (5 of 13). Adhesions were more frequent at a gestational age more than 8 weeks (24.4 vs 3.7\%; prevalence risk of 6.6) as well as in missed abortion (41.7 vs 12.1\% ; prevalence risk of 3.54). Conclusions: prevalence of intrauterine adhesions after manual vacuum aspiration was $16.3 \%$. Missed abortion and advanced gestational age seem to be associated with the occurrence of adhesions.

KEYWORDS: Adhesions; Uterine diseases; Abortion; Vacuum curettage/methods

\section{Introdução}

Aproximadamente $15 \%$ das gestações clinicamente reconheciveis terminam em abortamento. A evacuação uterina, comumente realizada no abortamento para evitar hemorragia e infecção, está associada a seqüelas reprodutivas significativas, tais como abortamentos de repetição, alterações menstru-

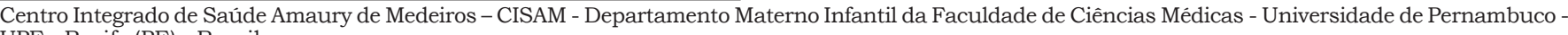
UPE - Recife (PE) - Brasil.

1 Médico Assistente do CISAM-Universidade de Pernambuco - UPE - Recife (PE) - Brasil.

2 Médica Assistente do CISAM-Universidade de Pernambuco - UPE - Recife (PE) - Brasil.

3 Acadêmio da Universidade de Pernambuco - UPE - Recife (PE) - Brasil.

4 Professor Regente da Disciplina de Tocoginecologia da Universidade de Pernambuco - UPE - Recife (PE) - Brasil.

Correspondência: Hélio de Lima Ferreira Fernandes Costa

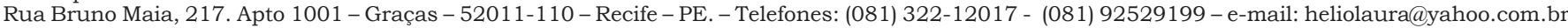


ais, infertilidade, parto prematuro e acretismo placentário ${ }^{1-4}$. Essas alterações são atribuídas, em sua grande maioria, à formação de aderências intra-uterinas (AIU) ou sinéquias, relacionadas com o traumatismo da camada basal do endométrio ocasionado pela curetagem uterina ${ }^{5}$.

Admite-se que a incidência de AIU seja elevada em mulheres submetidas à curetagem uterina $^{6}$, apesar de a maioria dos resultados dos estudos terem sido baseados em amostras populacionais com critérios de inclusão, formas clinicas de abortamento e oportunidade cirúrgica para esvaziamento uterino distintos ${ }^{7}$. Na tentativa de minimizar a ocorrência das AIU, outros métodos, como a aspiração manual a vácuo (AMV), vêm sendo cada vez mais empregados no tratamento dos abortamentos ${ }^{8-10}$. Este procedimento, além de simples, rápido e barato, apresenta taxas de complicações sérias com necessidade de internamento hospitalar tão baixas quanto $0,1 \%^{11-13}$. Recente meta-análise ${ }^{14}$, comparando a curetagem uterina convencional com a AMV no tratamento de abortamentos espontâneos, não encontrou diferenças entre os dois métodos no que se refere à perda de sangue, dor abdominal pós-operatória, evacuação uterina incompleta, morbidade febril, necessidade de re-hospitalização ou antibioticoterapia curativa.

A ocorrência de AIU é descrita como provável complicação da AMV, de etiologia ainda incerta $^{15}$, embora a dilatação cervical excessiva e o próprio trauma da sucção sejam cogitados como possiveis causas. Aventa-se que a AMV com cânulas de plástico teoricamente levaria a menor risco de formação de AIU que a curetagem convencional, com instrumental metálico ${ }^{16}$. Não obstante, a literatura carece de estudos que atestem esta vantagem.

Desde 1996, a AMV vem sendo rotineiramente empregada no Centro Integrado de Saúde Amaury de Medeiros (CISAM). O objetivo do presente estudo foi verificar a freqüência de AIU diagnosticadas por histeroscopia, 4 a 6 semanas após AMV, em mulheres internadas para tratamento de abortamento e caracterizar as AIU quanto à localização, constituição e gravidade, bem como tentar associar sua ocorrência com a forma clínica do abortamento, idade gestacional e passado cirúrgico sobre o útero.

\section{Métodos}

No período de novembro de 2000 a agosto de 2001, foi realizado estudo descritivo prospectivo do tipo corte transversal, com comparação de grupos em 102 mulheres internadas com diagnóstico de abortamento (incompleto, inevitável, infectado, retido ou molar) e submetidas ao esvaziamento uterino pela técnica de AMV. O diagnóstico da forma clínica do abortamento levou em consideração os dados clínicos, ultra-songráficos e histopatológicos. Todos os casos de abortamento ocorridos nos dias dos plantões do pesquisador principal e de um grupo de três pesquisadores auxiliares que se dispuseram a colaborar com o estudo foram considerados elegiveis. Foram considerados como critérios de exclusão o diagnóstico prévio de AIU e o uso de técnica para AMV diferente da padronizada. Foram excluídas da pesquisa as pacientes submetidas à AMV que desistiram ou não retornaram para realização da histeroscopia diagnóstica no prazo de 40 a 60 dia, as que fossem submetidas à histerectomia ou engravidassem após a AMV e antes da histeroscopia e aquelas com histeroscopia inconclusiva. Após serem esclarecidas verbalmente dos objetivos e etapas da pesquisa, as mulheres que concordaram em participar e que preencheram os critérios de inclusão assinaram termo de consentimento.

Os dados reprodutivos das pacientes foram coletados pelo pesquisador principal por meio de entrevista sistemática com todas as participantes. Os dados referentes à AMV foram coletados por meio de entrevista com a equipe médica responsável pelo procedimento e por meio de consulta aos prontuários de cada paciente. Os dados referentes aos achados histeroscópicos foram registrados diretamente pelo pesquisador que realizou todos os exames.

A AMV era realizada por médicos plantonistas e residentes do CISAM segundo técnica normatizada no serviço e descrita a seguir: com a paciente em posição de litotomia, eram realizados anti-sepsia da genitália externa e região perineal, toque vaginal (para avaliar tamanho e posição do útero), introdução do espéculo vaginal com visualização completa do colo uterino, bloqueio cervical com lidocaína a $2 \%$ sem vasoconstrictor, infiltrando-se 2 a $4 \mathrm{~mL}$ às 5 e 7 horas, pinçamento do colo com Pozzi e histerometria da cavidade uterina. O próximo passo era a aspiração manual a vácuo, que consistiu na retirada do conteúdo uterino por sucção, mediante transferência de vácuo produzido em seringa de $60 \mathrm{~mL}$ (seringa de Karman), conectada a cânulas plásticas cujos diâmetros variaram de 4 a $12 \mathrm{~mm}$, selecionadas de acordo com o diâmetro do colo. Quando o colo não permitia a penetração da cânula com o menor diâmetro disponivel, realizava-se a dilatação cervi- 
cal com velas de Hegar. O procedimento era realizado por movimento de vai-e-vem da cânula e dado como concluído quando da observação de sangue vermelho-vivo, espumoso, fluindo do canal cervical, bem como pela percepção da limitação dos movimentos da cânula apreendida pelas paredes uterinas na presença de vácuo.

Por ocasião da alta hospitalar, era agendado o retorno da paciente num prazo de 40 a 60 dias após a AMV, ocasião em que ela era submetida a nova entrevista e histeroscopia, ambos com o pesquisador principal.

A histeroscopia foi realizada no setor de endoscopia ginecológica do CISAM, com auxílio de endoscópio rígido e insuflador uterino de gás carbônico $\left(\mathrm{CO}_{2}\right)$, realizando-se sedação com meperidina excepcionalmente, quando necessário. A técnica do exame seguiu a padronização estabelecida no consenso brasileiro em vídeo-endoscopia ginecológica ${ }^{18}$. Os achados histeroscópicos considerados anormais, como, por exemplo, aderências e restos ovulares, eram gravados em fita cassete e posteriormente apresentados a outro pesquisador para respaldar o diagnóstico.

A histeroscopia seria considerada inconclusiva quando não fosse possivel visualizar a cavidade endometrial em virtude de sangramentos ou qualquer outro achado, excentuando-se as AIU. Não houve registro de nenhum laudo inconclusivo neste estudo.

A análise dos dados foi realizada no software de domínio público Epi-Info, versão 6.04. Empregou-se o teste exato de Fisher e o cálculo da razão de prevalência (RP) para testar a associação entre a ocorrência de AIU e as variáveis: idade gestacional, tipo de abortamento e cirurgias sobre o útero. Utilizou-se ANOVA para testar a associação entre a idade da paciente e a ocorrência de AIU. Adotouse $5 \%$ como nível de significância.

O presente estudo foi aprovado pelo Comitê de Ética do CISAM antes do início da coleta dos dados, sendo garantido o sigilo das informações e o cumprimento dos preceitos contidos na Declaração de Helsinque.

\section{Resultados}

Um total de 102 pacientes foi incluído no estudo. Destas, 80 retornaram para a realização da histeroscopia e $22(21,5 \%)$ foram excluídas, todas por não comparecimento à histeroscopia. Não houve casos de histeroscopia inconclusiva, nem tampouco gravidez ou histerectomia entre a AMV e a histeroscopia. Foram encontradas AIU em 13 das 80 mulheres que concluíram a pesquisa, resultando em prevalência de $16,3 \%$.

Além das AIU, as únicas alterações intrauterinas observadas foram um caso de pólipo endometrial e um de deposição de fibrina no endométrio. Esvaziamento incompleto da cavidade uterina, com necessidade de curetagem após a AMV, foi descrito em duas mulheres, sendo observada presença de AIU à histeroscopia em apenas uma delas. Apenas uma mulher referiu dor e/ou febre nos primeiros dias após a AMV, não relacionadas à AIU até o momento da realização da histeroscopia.

As pacientes tinham idade entre 15 e 48 anos, com média de 25,6 $\pm 7,1$ anos. A média de idade das pacientes que apresentaram AIU foi de $28,5 \pm 9,3$ anos, ao passo que as que não apresentaram AIU tinham idade média de $25,1 \pm 6,5$ anos, não se observando diferença significante entre os dois grupos no que se referiu à idade $(p=0,12)$.

$\mathrm{Na}$ Tabela 1 encontram-se distribuídos os casos de AIU segundo suas características principais. Em 11 das 13 pacientes acometidas as aderências eram de gravidade mínima, sendo nove delas de constituição mucosa. A localização mais freqüente foi a lateral, presente em cinco mulheres. Das quatro pacientes com aderências fibrosas, três tinham sido submetidas a procedimentos cirúrgicos prévios sobre o útero (uma delas havia tido parto a fórcipe e cesárea, outra havia tido cesárea e curetagem uterina e a terceira havia tido curetagem com perfuração uterina e histerorrafia).

Tabela 1 - Distribuição das aderências intra-uterinas segundo sua constituição, localização e gravidade, Cisam - Recife, 2000-2001.

\begin{tabular}{lcc}
\hline Características das AIU & $\mathbf{n}$ & $\%$ \\
\hline Constituição & 4 & 30,8 \\
Fibrosa & 9 & 69,2 \\
Mucosa & & \\
$\begin{array}{l}\text { Localização* } \\
\text { Cervical/Ístmica }\end{array}$ & 3 & 23,1 \\
Central & 3 & 23,1 \\
Lateral & 5 & 38,5 \\
Fúndica & - & 0 \\
Cornual & 3 & 23,1 \\
Gravidade & & 84,6 \\
Mínima & 11 & 0 \\
Moderada & - & 13,4 \\
Grave & 2 & \\
\hline
\end{tabular}

AIU - Aderências intra-uterinas.

*Uma mesma paciente apresentou duas localizações diferentes. 
Ao analisarmos a prevalência de AIU, de acordo com a idade gestacional por ocasião da AMV (Tabela 2), observamos que $24,4 \%$ das pacientes com abortamento posterior à oitava semana apresentaram AIU, enquanto que apenas 3,7\% das que abortaram até a oitava semana desenvolveram a condição (risco de prevalência $=6,6 ; p=0,02$ ).

Tabela 2 - Distribuição das mulheres submetidas à aspiração manual a vácuo segundo a idade gestacional e a presença de aderências intra-uterinas, Cisam - Recife, 2000-2001.

\begin{tabular}{lccc}
\hline & \multicolumn{3}{c}{ Idade gestacional } \\
.AIU & $\leq 8$ semanas & $>8$ semanas & Total \\
\hline Sim & $1(3,7 \%)$ & $11(24,4 \%)$ & $12(16,7 \%)$ \\
Não & $26(96,3 \%)$ & $34(75,6 \%)$ & $60(83,3 \%)$ \\
Total & $27(100 \%)$ & $45(100 \%)$ & $* 72(100 \%)$
\end{tabular}

AIU - Aderências intra-uterinas.

$p=0,02$ (Fisher).

$\mathrm{RP}=6,6$ (IC: 0,9 a 48,3 ).

*8 pacientes não sabiam a data da última menstruação.

Quanto à forma clínica do abortamento, encontramos maior prevalência de AIU nas pacientes com abortamento retido (5 entre 12 ) do que entre aquelas com abortamento incompleto/inevitável (8 das 66 mulheres), com risco de prevalência igual a 3,54 $(\mathrm{p}=0,02)$, conforme apresentado na Tabela 3.

Tabela 3 - Distribuição das mulheres submetidas à aspiração manual a vácuo segundo a forma clínica do abortamento e a presença de aderências intra-uterinas, Cisam - Recife, 2000-2001.

\begin{tabular}{lccc}
\hline & \multicolumn{2}{c}{ AIU } & \\
Tipos de abortamento & Sim & Não & Total \\
\hline Incompleto/inevitável & $8(12,1 \%)$ & $58(87,9 \%)$ & $66(100,0 \%)$ \\
Retido & $5(41,7 \%)$ & $7(58,3 \%)$ & $12(100,0 \%)$ \\
Molar & - & $1(100,0 \%)$ & $1(100,0 \%)$ \\
Infectado & - & $1(100,0 \%)$ & $1(100,0 \%)$ \\
Total & $13(16,3 \%)$ & $67(83,7 \%)$ & $80(100,0 \%)$
\end{tabular}

AIU - Aderências intra-uterinas.

$\mathrm{p}=0,02{\text { (Fisher })^{*}}^{*}$

$\mathrm{RP}=3,54($ IC95\% $=1,39-9,01)$.

*Comparação entre as formas de abortamento incompleto/inevitável e retido.

A Tabela 4 explicita a ocorrência de AIU de acordo com os antecedentes de procedimentos cirúrgicos sobre o útero (cesarianas e curetagens). Comparando-se o grupo das pacientes submetidas a qualquer tipo de cirurgia com o grupo daquelas sem cirurgias prévias, não se observou diferença significante na ocorrência de AIU (20,7 vs 13,7\%). Das 6 pacientes submetidas a curetagem e cesarianas, $3(50 \%)$ tiveram AIU, configurando diferença de significância limítrofe com as demais pacientes $(p=0,05)$.
Tabela 4 - Distribuição das pacientes submetidas à aspiração manual a vácuo de acordo com o passado de cirurgia sobre o útero e a ocorrência de aderências intra-uterinas, Cisam - Recife, 2000-2001.

\begin{tabular}{lrrrrrrrr}
\hline Cirurgia & \multicolumn{4}{c}{ AIU } & & & \multicolumn{1}{c}{ p (Fisher) } \\
& \multicolumn{2}{c}{ Sim } & \multicolumn{2}{c}{ Não } & \multicolumn{2}{c}{ Total } & \\
& $\mathbf{n}$ & $\%$ & $\mathbf{n}$ & $\%$ & $\mathbf{n}$ & $\%$ & \\
\hline Sim & 6 & 20,7 & 23 & 79,3 & 29 & 100,0 & 0,30 \\
Curetagem & 1 & 8,3 & 11 & 91,7 & 12 & 100,0 & 0,37 \\
Cesárea & 2 & 18,2 & 9 & 81,8 & 11 & 100,0 & 0,56 \\
Curetagem+cesárea* & 3 & 50,0 & 3 & 50,0 & 6 & 100,0 & 0,05 \\
Não & 7 & 13,7 & 44 & 86,3 & 51 & 100,0 & - \\
Total & 13 & 16,3 & 67 & 83,7 & 80 & 100,0 & - \\
\hline
\end{tabular}

AIU - Aderências intra-uterinas.

$p=0,32$ (todas as pacientes submetidas a curetagem vs não operadas).

$p=0,10$ (todas as pacientes submetidas a cesárea vs não operadas).

*Uma das pacientes foi submetida a miorrafia após perfuração uterina na curetagem.

\section{Discussão}

A prevalência de AIU observada em nosso estudo situou-se dentro da faixa de 15 a $19 \%$ referida na literatura após esvaziamento uterino com método cirúrgico ${ }^{7}$. É plausivel que o resultado possa estar superestimado por não ter sido realizada histeroscopia prévia à AMV para excluir as paciente com AIU anterior ao abortamento em questão. De fato, em quatro pacientes foram encontradas aderências fibrosas, que se desenvolvem mais freqüentemente após um ano ou mais decorridos da lesão ${ }^{7}$ e que talvez não possam ser atribuídas à AMV em questão. Corrobora esta hipótese o fato de três das quatro pacientes com AIU fibrosas terem tido procedimento cirúrgico prévio sobre o útero. Se considerarmos, portanto, apenas as aderências mucosas, a prevalência seria de 11,2\% (nove em 80 pacientes).

A limitação do período de tempo para realizar a histeroscopia, entre 40 e 60 dias póscuretagem, objetivou aguardar a regressão das alterações induzidas pelo processo gestacional, de forma a minimizar a possibilidade de encontrarmos sangue, restos ovulares ou dilatação residual do colo uterino que impedissem a adequada visualização da cavidade endometrial. De fato, nenhuma das situações acima foi verificada. Ademais, o curto intervalo entre a curetagem e a histeroscopia permitiu diferenciar as AIU presumivelmente recentes das tardias.

Apesar do predomínio de casos de abortamento incompleto/inevitável no estudo, a prevalência de AIU no grupo de mulheres com abortamento retido foi 3,5 vezes maior que naquelas. Outros autore ${ }^{20}$ também encontraram maior prevalência de AIU, diagnosticada por histerografia, após abortamento retido. Para $\mathrm{March}^{5}$, o material ovular 
necrosado, retido na cavidade endometrial, induziria uma resposta inflamatória, com maior atividade fibroblástica e formação de colágeno, antes do processo de regeneração endometrial se completar.

Os locais de maior ocorrência das AIU foram as paredes laterais da cavidade endometrial. $\mathrm{O}$ pequeno tamanho amostral não nos permitiu tirar conclusões definitivas quanto à razão da ausência de AIU na região fúndica. Não obstante, seria atraente supor que a forma romba da extremidade da cânula de AMV, bem como a disposição lateral de suas fenestras, poderiam reduzir o traumatismo endometrial no fundo uterino, reduzindo um dos principais fatores envolvidos na etiopatogenia da doença ${ }^{15,19}$.

Tal como Romer ${ }^{20}$, também não encontramos diferença entre os grupos com e sem aderências em relação à idade média das mulheres analisadas. Teoricamente, esperar-se-ia maior prevalência de AIU em torno dos 30 anos de idade, quando a maioria das mulheres já teria experimentado eventos obstétricos, como partos e abortamentos, além de cirurgias sobre o útero. A análise do fator idade em nossa amostra foi, em grande parte, influenciada pelo fato de todas as mulheres terem sido recrutadas logo após o episódio de abortamento, estando necessariamente em periodo reprodutivo. Para uma real estimativa da prevalência de AIU nas distintas faixas etárias, seria necessário rastreamento histeroscópico populacional, com importantes implicações éticas.

O predomínio de AIU no grupo de mulheres com idade gestacional maior que 8 semanas provavelmente se deve ao fato de que, pelo maior tamanho e conteúdo ovular, estes úteros necessitariam de maior tempo de aspiração para serem completamente esvaziados. Como conseqüência, seriam submetidos a um traumatismo endometrial proporcionalmente maior. Ademais, o maior conteúdo intra-uterino também poderia predispor ao esvaziamento incompleto da cavidade, como observado em duas de nossas pacientes, ambas com idade gestacional superior a 12 semanas e que tiveram que ser submetidas à curetagem uterina convencional após a AMV. Além disso, no período pós-abortamento em gestações mais avançadas, as pacientes estariam expostas a um hipoestrogenismo mais prolongado que retardaria a regeneração endometrial e facilitaria a formação das AIU ${ }^{21}$.

$\mathrm{Na}$ presente amostra, 29 mulheres tinham passado cirúrgico uterino. Ao contrário de outros autores $^{15,20}$, não encontramos associação entre a realização de curetagens prévias e a ocorrência de AIU $(p=0,32)$. Não podemos descartar a possibi- lidade de a aspiração intra-uterina ter destruído as aderências antigas, funcionando, paradoxalmente, como tratamento das mesmas. Finalmente, devemos considerar que a seleção de pacientes no período pós-abortamento pode excluir aquelas com aderências, determinadas por cirurgias prévias, que tenham levado à esterilidade. As pacientes submetidas a curetagem e cesárea tiveram prevalência de AIU de 50\%. A diferença, no limite da significância em relação às demais pacientes, sugere que o reduzido tamanho amostral pode ter mascarado uma associação porventura existente.

Para evitarmos viés de suspeita diagnóstica, não foram considerados como critérios de seleção para admissão na pesquisa aspectos como: forma clinica de abortamento, idade, paridade ou antecedentes cirúrgicos.

Os resultados do presente estudo, em que pese as limitações de um estudo descritivo, não reforçam a hipótese de ser a AMV método com menor potencial de provocar aderências intrauterinas quando comparada com a curetagem metálica, tendo em vista uma prevalência semelhante à observada na literatura para o método de referência. Parecem ser fatores associados ao surgimento das AIU a idade gestacional avançada e o aborto retido. Novas pesquisas, preferencialmente com o desenho de ensaio clínico randomizado controlado, comparando os dois métodos, poderão esclarecer esta relevante questão da assistência obstétrica ao abortamento.

\section{Referências}

1. Traina E, Mattar R, Moron AF, Albuquerque Neto LC, Mateus ED. Acurácia diagnóstica da histerossalpingografia e da ultra-sonografia para avaliação de doenças da cavidade uterina em pacientes com abortamento recorrente. Rev Bras Ginecol Obstet. 2004;26(7):527-33.

2. Ozumba B, Ezegwui H. Intrauterine adhesions in an African population. Int $\mathrm{J}$ Gynaecol Obstet. 2002;77(1):37-8.

3. Katz Z, Ben-Arie A, Lurie S, Manor M, Insler V. Reproductive outcome following hysteroscopic adhesiolysis in Asherman's syndome. Int $\mathrm{J}$ Fertil Menopausal Stud. 1996;41(5):462-5.

4. Diamond MP, Freeman ML. Clinical implications of postsurgical adhesions. Hum Reprod Update. 2001;7(6):567-76.

5. March CM. Intrauterine adhesions. Obstet Gynecol Clin North Am. 1995;22(3):491-505. 
6. Golan A, Schneider D, Avrech O, Raziel A, Bukovsky I, Caspi E. Hysteroscopic findings after missed abortion. Fertil Steril. 1992;58(3):508-10.

7. Kulier R, Fekih A, Hofmeyr GJ, Campana A. Surgical methods for first trimester termination of pregnancy. Cochrane Database Syst Rev. 2001;(4):CD002900.

8. Dalton V, Castleman L. Manual vacuum aspiration for treatment of early pregnancy loss. Postgrad Obstet Gynecol. 2002;22(19):1-5.

9. Holanda AAR, Freire HP, Santos D, Barbosa MF, Barreto CFB, Felinto AS, et al. Tratamento do abortamento do primeiro trimestre da gestação: curetagem versus aspiração manual a vácuo. Rev Bras Ginecol Obstet. 2003;25(4):271-6.

10. Westfall JM, Sophocles A, Burggraf H, Ellis S. Manual vacuum aspiration for first-trimester abortion. Arch Fam Med. 1998;7(6):559-62.

11. Fonseca W, Misago C, Fernandes L, Correia L, Silveira D. Uso da aspiração manual a vácuo na redução do custo e duração do internamento por abortamento incompleto em Fortaleza, CE, Brasil. Rev Saúde Pública. 1997;31(5):472-8.

12. Hemlin J, Moller B. Manual vacuum aspiration, a safe and effective alternative in early pregnancy termination. Acta Obstet Gynecol Scand. 2001;80(6):563-7.

13. Foster-Rosales A, Koontz SL, Molina de Perez O, Leon $\mathrm{K}$. Cost savings of manual vacuum aspiration for endometrial sampling in El Salvador. Contraception. 2003;68(5):353-7.

14. Say L, Kulier R, Gülmezoglu M, Campana A. Medical versus surgical methods for first trimester termination of pregnancy. Cochrane Database Syst Rev. 2005;(2):CD 003037.
15. Friedler S, Margalioth EJ, Kafka I, Yaffe H. Incidence of post abortion intrauterine adhesions evaluated by hysteroscopy: a prospective study. Human Reprod. 1993;8(3):442-4.

16.Lukman HY, Pogharian D. Management of incomplete abortion with manual vacuum aspiration in comparison to sharp metallic curette in an Ethiopian setting. East Afr Med J. 1996;73(9):598603.

17. Hamou J, Salat-Baroux J, Siegler AM. Diagnosis and treatment of intrauterine adhesions by microhysteroscopy. Fertil Steril. 1983;39(3):321-6.

18.Adoni A, Palti Z, Milwidsky A, Dolberg M. The incidence of intrauterine adhesions following spontaneous abortion. Int J Fertil. 1982;27(2):1178.

19. Rocha Cortes F, Chacon Iñiguez J, Amaro Patron $\mathrm{R}$, Ramon Alvarez Morales J, Vargas Zepeda D. Aspiración manual endouterina com jeringa de Karman (AMEU). Estudio multicentrico em Sonora, Sinaloa, Mexico. Ginecol Obstet Mex. 1996;64:97104.

20. Romer T. Post-abortion hysteroscopy: a method for early diagnosis of congenital and acquired intrauterine causes of abortion. Eur J Obstet Gynecol Reprod Biol. 1994;57(3):171-3.

21.Billings DL, Fuentes Velásquez J, Perez-Cuevas R. Comparing the quality of three models of postabortion care in public hospitals in Mexico City. Int Fam Plan Perspect. 2003;29(3):112-20. 